\title{
COMPUTATIONAL ANALYSES OF SMALL ENDOSSEOUS IMPLANTS IN OSTEOPOROTIC BONE
}

\author{
A.J. Wirth ${ }^{1}$, R. Müller ${ }^{1}$, and G.H. van Lenthe ${ }^{1,2, *}$ \\ ${ }^{1}$ Institute for Biomechanics, ETH Zurich, Zurich, Switzerland \\ ${ }^{2}$ Division of Biomechanics and Engineering Design, K.U.Leuven, Leuven, Belgium
}

\begin{abstract}
For many years orthopedic implants were developed for patients with good bone stock. Recently it has become clear that these implants have a decreased performance when implanted in bone with low density, such as in osteoporosis. Reduced performance in osteoporotic bone is not unexpected because of the reduced quality of the peri-implant bone and the reduced bone-implant contact area. Nevertheless, the precise failure mechanisms are not well understood. Although experimental testing is considered the gold standard to determine implant fixation, it is hampered by many limitations. Computational models could potentially aid in obtaining a better understanding of implant fixation as they allow analyzing the mechanical interaction between implants and peri-implant tissues. This article provides a review of the existing finite element models of small endosseous implants in bone. The aim is to analyze the potential of such models to aid the understanding of implant failure mechanisms with the goal of improving implant performance in low quality bone.
\end{abstract}

Keywords: Finite element analysis, endosseous implants, dentistry, orthopedics, peri-implant bone.

* Address for correspondence:

G. Harry van Lenthe

Institute for Biomechanics

ETH Zurich, HPI F22

Wolfgang-Pauli-Strasse 14

CH-8093 Zurich, Switzerland

Telephone Number: +41.44.632.4591

FAX Number: +41.44 .633 .1573$

E-mail: vanlenthe@ethz.ch

URL: http://www.biomech.ethz.ch
Introduction

Small endosseous implants are widespread and important components of modern orthopedics and dentistry. They are implanted with their main part penetrating trabecular and cortical bone. This is in contrast with larger implants, such as hip joint endoprostheses, which are generally implanted in the medullary cavity of long bones. Despite the success small threaded endosseous implants have achieved in modern orthopedic and dental medicine, there are still cases in which they do not perform well. Biomechanical studies have shown that prevailing small endosseous implants show decreased performance when implanted into bone with low density, such as evoked by osteoporosis (Bonnaire et al., 2005; Koistinen et al., 2005; Seebeck et al., 2004). It appears evident that there is potential to enhance implant performance. In order to develop new implant designs for low quality bone, a proper investigation of the mechanisms behind decreased implant performance is crucial. In the last decades a high number of studies that analyzed small endosseous implants in bone constructs using finite element analysis (FEA) have been published (references included in the next sections). These studies have analyzed different bones and implants with largely varying computational models. The aim of the present work is to review these publications and analyze if, and how, finite element analysis can be used to study the suboptimal performance of small endosseous implants in low-quality bone.

\section{Implant Failure in Osteoporotic Bone}

The emergence of high numbers of patients with low bone quality, as caused by osteoporosis, has led to a new problem area in orthopedics (Kanis and Johnell, 2005). Osteoporosis is a disease which is characterized by a structural deterioration of bone architecture and a general decrease in bone mass (Fig. 1) (Consensus Development Conference, 1993). This decrease in bone quality reduces the ability of bone to withstand mechanical loads and thus increases the risk of fracture. Osteoporosis is not limited to specific sites; nevertheless, the distal radius, proximal femur and vertebrae are responsible for almost all osteoporosis-induced fractures (Burge et al., 2007). The bones at these sites have a dense outer shell called cortical bone, and a relatively high fraction of complex sponge-like inner structures called trabecular or cancellous bone (Fig. 2). Despite of research for decades, the precise mechanisms involved in the development of osteoporosis, as well as the optimal way of preventing it, are not completely understood (Boonen et al., 2008; Kesson et al., 1947). It is 

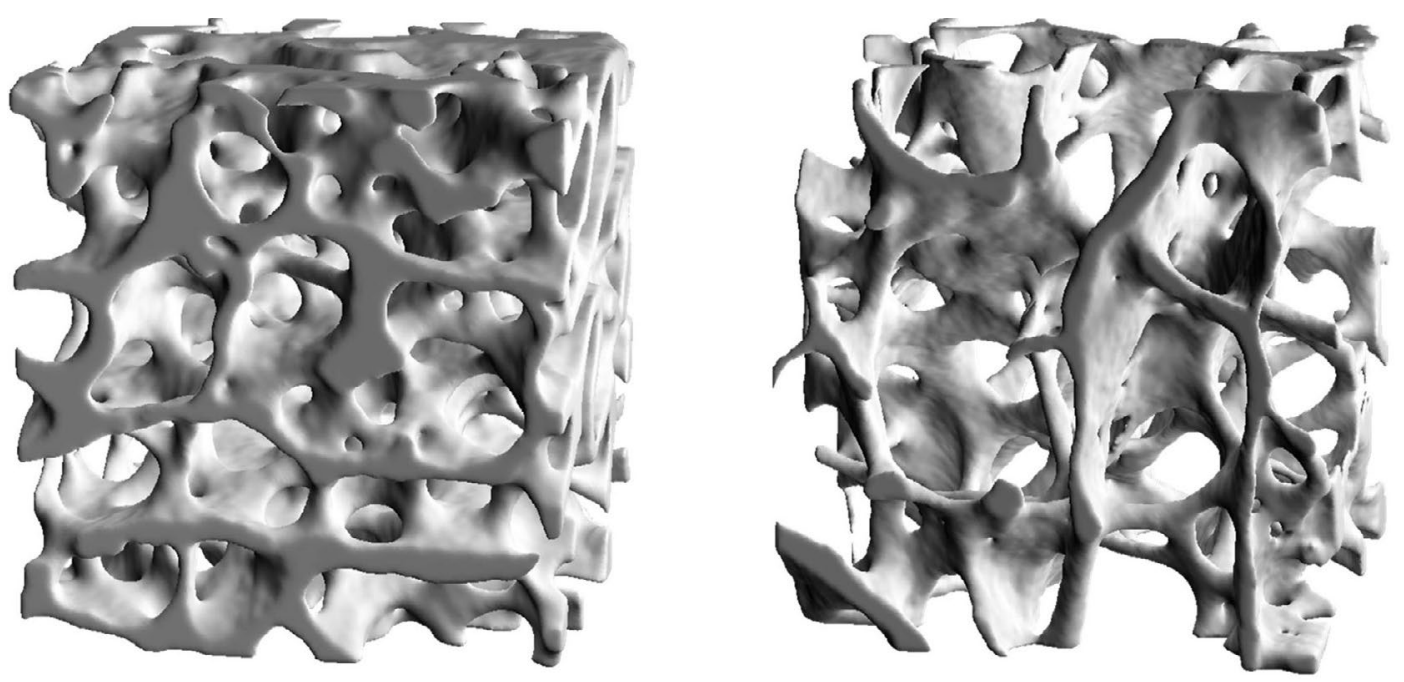

Fig. 1. MicroCT images of trabecular bone microstructure in young and healthy (left) and osteoporotic (right) femoral head. Scale line $1 \mathrm{~mm}$.

estimated that half of women and a quarter of men aged 50 years or older will suffer a fracture related to osteoporosis within their remaining lifetime (Chrischilles et al., 1991). Thus, osteoporotic fractures have become a major burden on public healthcare systems. Moreover, due to the ageing population in developed countries and an insufficient prevention of osteoporosis, the number of osteoporotic fractures is expected to increase significantly within the coming years, as are the costs resulting from them (Burge et al., 2007; Kanis and Johnell, 2005; Ray et al., 1997).

For many years, orthopedic implants were developed for patients with good bone stock (Schneider et al., 2005). In recent years it has become clear that these implants have a decreased performance when implanted into osteoporotic bone. Several approaches exist to treat fractures of bone with low quality, including the augmentation of peri-implant bone with bone cement (Frankel et al., 2007). Another common approach is using a high number of screws and multiple plates. On one hand such systems provide increased stability due to interlocking and redundancy; however, at the same time, they introduce defects into the bone which potentially reduce its mechanical competence and consequently also the stability and strength of the whole fixation (Scott et al., 1985).

Probably the most important cause for implant failure by number of cases is low bone quality. Other circumstances can also evoke implant failure, such as overloading, incomplete fracture reduction and inappropriate osteosynthesis as caused by non-optimal choice of implants by the surgeon. A proper selection of implants is hampered by the fact that no gold standards exist. Hence the optimal implant, combining structural stability with reliable anchorage in bone, is yet not known. In order to develop new designs for low quality bone, a proper investigation into the mechanisms behind this decreased implant performance would therefore be crucial. However, the precise nature of these failure mechanisms is as yet unknown.

A further complication in the analysis of implant failure is the difficulty of detecting failed implants in clinical

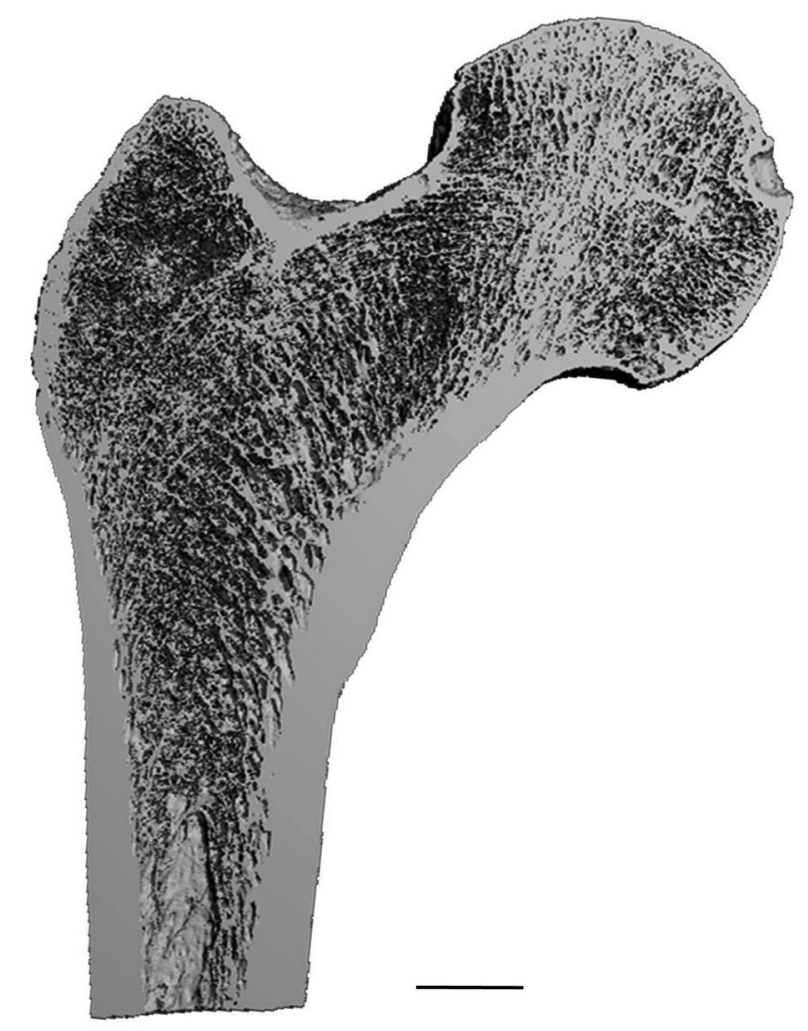

Fig. 2. Porous trabecular and dense cortical bone in a proximal human femur as assessed by high-resolution computed tomography (image courtesy of T. L. Mueller, Institute for Biomechanics, ETH Zurich, Zurich, Switzerland). Scale line $10 \mathrm{~mm}$.

practice; hence the total failure rate is hard to estimate. Implant failure is often only detected after the whole healing process has turned out to be suboptimal, or even only after explantations. In the latter case it is not known whether an implant actually fulfilled its task $a b$ initio. Failed implants can lead to a loosening of dental prostheses, which may cause inflammations and necrosis, or in the case of orthopedic trauma implants, to suboptimal fracture heal- 
ing. However, even if primary stability is provided, small endosseous implants can still fail in the mid- or long-term. In addition to the difficulties in detecting implant failure there may be other factors that limit the publication of proper statistics on implant failure, ranging from patients' and surgeons' data protection to the possible economic and legal interests of implant producers and clinics.

\section{Assessment of Implant Fixation}

\section{Experimental testing}

Experimental testing is considered the gold standard to determine implant fixation. However, performing experimental biomechanical measurements on patients is almost impossible, as not all mechanical aspects can be investigated by experimental measurements, for example no methods exist to measure the forces acting on a screw in vivo. Actually, implant fixation strength can only be tested in cadaveric bone because it is a destructive test. Unfortunately, access to cadaveric osteoporotic human bone is generally very limited, which restricts the use of standardized tests and the elaboration of statistics. Sheep, one of the standard animal models used for testing human orthopedic implants in vivo, do not suffer from any systemic disease resulting in bone of low quality that can be compared to that found in human patients suffering from osteoporosis. Only in recent years, following many unsuccessful endeavors, has it become possible to establish artificial osteoporotic sheep models (Egermann et al., 2008; Zarrinkalam et al., 2008), but due to ethical and financial reasons, such large animal models are not appropriate for an early-stage development of new orthopedic implants. Furthermore, large animal models may still be influenced by external factors in need of further investigation, and more expertise must be gained with these models before they can be used in implant fixation studies (Arens et al., 2007). As an alternative animal model for very small implants, the rat has successfully been used to test anchorage of implants in low density bone (Gabet et al., 2006).

\section{Computational analyses}

Because of the limitations in experimental testing of bone-implant failure, alternatives have been sought. Engineering principles are fundamental in orthopedics and were already applied by Carl Hansmann, the son of an engineer, for the design of the first small threaded endosseous implants (Wolter and Jürgens, 2006). Primary implant stability can be predicted by means of analytical formulas (Firoozbakhsh et al., 1994), but despite giving good predictions for artificial bone, such formulas have not yet been validated for real bone and probably no longer hold true if high variations in local bone quality and microarchitectural orientation are present. Computer simulations which incorporate engineering principles are a highly accepted tool in modern engineering in the analysis and optimization of the mechanical competence of structures. One such tool, which has also been successfully introduced into orthopedics, is the finite element method, also called finite element analysis (FEM resp. FEA). FEA is a numerical technique for solving boundary-value problems. The basic concept is to find a solution of the differential equation of a field problem by numerical means. Therefore, the complex area is divided into a high, but finite, number of smaller and much simpler partial regions, called elements. For these elements an approximation function for the unknown variables is chosen. Then the approximate function for the whole region is composed of the approximate functions of the partial regions and the resulting algebraic system is solved numerically by applying an optimizing algorithm. While the main fundamental concepts for FEA were already established by Courant in 1942 and Turner et al. in 1956 (Gupta and Meek, 1996), its practical usage was very restricted due to the limited computational power available at that time. FEA achieved its breakthrough beyond aeronautics and defense technology in the early 1970s when, for the first time, it was also used for the analysis of bones (Rybicki et al., 1972). This took place in an era of enormous progress in endosseous implant design in dentistry, mainly driven by the groundbreaking work of Per-Ingvar Brånemark (Brånemark et al., 1969). It was therefore no coincidence that in 1973 the presumably first FE model of a small endosseous implant was published analyzing a dental implant (Tesk and Widera, 1973). Since then, FE analysis has become a well-established technique and has emerged into many other fields of bone-related research, among them also the field of small endosseous implants in orthopedics. A search in the MEDLINE database with the keywords "finite element" in combination with "bone" resulted in 16 publications from the 1970s, 162 from the 1980 s, 575 from the 1990s and 2026 from the 2000s, demonstrating a strongly increased usage of FEA.

\section{Main Requirements for Finite Element Analysis of Bone-Implant Constructs}

The proper modeling of bones and implants for FEA includes three important aspects which can easily be expanded to bone-implant constructs:

i. an adequate representation of bone and implant geometry;

ii. an adequate representation of bone and implant material properties;

iii. an appropriate description of the boundary

conditions, i.e. what are the directions and

magnitudes of the forces and displacements acting

on the construct.

The presence of implants leads to further requirements, most importantly:

iv. an accurate representation of bone-implant interaction;

v. an appropriate representation of failure behavior in bone-implant constructs.

These five aspects will be discussed in more detail below in conjunction with the modeling approaches that have been presented in the literature.

\section{Bone and implant geometry}

The bone geometry of elderly people who typically suffer from osteoporotic fractures varies greatly from that of younger people (Beck et al., 1993) and shows high in- 
ter-specimen variability. Therefore, patient-specific, or at least target group specific, models of bone geometry are necessary in FEA. Simple geometrical entities for conceptual studies can easily be generated using commercial pre-processors of FEA packages or computer aided design (CAD) programs; more realistic models of bone geometry are much more challenging because of the relatively complex geometries of bones. This especially holds true if besides the irregular external shape also the inner micro-architecture is to be modeled (Van Lenthe and Müller, 2006).

Micro-computed tomography has become the most widely used non-destructive imaging system for bone (Müller, 2009). It allows for a three-dimensional (3D) assessment of bone microstructure, with high accuracy and efficiency; some systems can even be used in vivo (Morgan and Bouxsein, 2005; Müller, 2002; Ploeg et al., 2004; Van Lenthe and Müller, 2006; Viceconti et al., 2004). The conversion of such images into FE models is usually done using (semi-) automatic meshing algorithms. Commercially available programs that allow such (near) automated conversion have been used for some studies for bone (Baggi et al., 2008; Lang et al., 2003).

Alternatively, bone geometry can be obtained from public domain databases which offer standardized models (Chen et al., 2004; Sowmianarayanan et al., 2008). However, access to osteoporotic bone models is still very limited in these databases. Moreover, current databases do not contain models that include bone microarchitecture. Perhaps further research will make it possible to identify the pathological changes in bone geometry which are responsible for a high number of osteoporotic fractures. This knowledge may help to establish standard osteoporotic bone models. However, as long as no such standards exist, it seems reasonable to generate finite element models with small endosseous implants from high-resolution CT images of bones belonging to the target-group.

In modeling trabecular bone two approaches have mainly been followed. In the first approach, bone is modeled as a continuum material without any geometrical porosity. The material properties are assumed to be isotropic, meaning that it behaves identical in all directions. In the second approach, care is taken to incorporate the detailed micro-architecture which includes the actual porous trabecular network. This has the advantage that the load-transfer mechanisms can be represented in much more detail. Another advantage is that the direction-dependent, anisotropic, behavior of the bone tissue is automatically taken into account.

It seems obvious that for a proper analysis of bone-implant interaction, and in order to include bone heterogeneity and anisotropy, one should model trabecular bone as a porous material with a specific microarchitecture. Although this guideline was actually set up for dental implants by Patra et al. (Patra et al., 1998), as cited by Van Staden et al. (Van Staden et al., 2006), it can also be applied for orthopedic implants. Nevertheless, almost none of the presented models included trabecular microstructure which was modeled as a continuum, distinguished from cortical bone only by different apparent material properties. The decision for the continuum approach was mainly motivated by the very large computational costs that arise when trabecular networks have to be modeled. In order to model trabeculae accurately, a high number of additional finite elements is needed compared to that which is necessary when trabecular bone is modeled as a continuum. Actually, most commercial finite element packages have so far not been able to solve models of appropriate size including microstructure bone. A model of a small endosseous implant in discrete trabecular structures was presented by Tsubota et al. (Tsubota et al., 2003) who used it to simulate bone remodeling. Such models can be solved using dedicated numerical packages, especially designed for this kind of model. Compared to commercial FE solvers, such solvers typically are restricted to many simplifications regarding the models, but in return allow analyzing geometrically highly detailed models (Arbenz et al., 2007).

The presence of implants leads to further requirements for the FE models. Most importantly, the implant geometry has to be represented accurately. The most direct way to obtain implant geometry is from CAD. This is feasible if the study is in silico only and a computational model of the implant is available (Genna et al., 2003), but it can become quite challenging if the design of a commercial implant is needed, unless information about geometry is provided by the manufacturer (Lang et al., 2003). In order to build up a computational model of such an implant, Baggi et al. (Baggi et al., 2008) used a comparative technique involving high-resolution pictures of actual implants. The accuracy of such a model, however, is hard to estimate. An alternative to such an approach was presented by Tsumita et al. (Tsumita et al., 2008), who used micro-computed tomography data which finally was converted into initial graphics exchange specifications format (IGES), which can be imported into most commercial CAD and FEA packages.

A complicating factor in generating models of bone-implant constructs is that the exact position and shape of the implants post op can be difficult to determine, for instance when during surgery the shape of plates has been adapted to fit the bone. In principle, several 3d imaging technologies, such as micro-computed tomography $(\mu \mathrm{CT})$ and in vivo peripheral quantitative computed tomography (pQCT) allow visualizing the exact implant location. However, most tube-based CT systems cannot determine the precise bone-implant interface characteristics. Furthermore, image quality is often hampered by metal-induced artifacts around the implants. Such artifacts preclude a proper measurement of the peri-implant bone (Lewitt and Bates, 1978; Williamson et al., 2002).

\section{Bone and implant material properties}

Most osteoporotic fractures occur in regions with a high content of trabecular bone, hence, the relatively complex material properties (Lenaerts and Van Lenthe, 2009; Morgan and Bouxsein, 2005) of bone have to be modeled adequately. The material properties of trabecular bone are strongly anisotropic, caused by a preferential orientation of the trabeculae. When modeling trabecular bone as a continuum material, the concept of the fabric tensor (Cowin, 1985; Gomez-Benito et al., 2005) can be used. The fabric tensor represents the main orientation of the trabeculae. Unfortunately, there is still no common agreement on how 

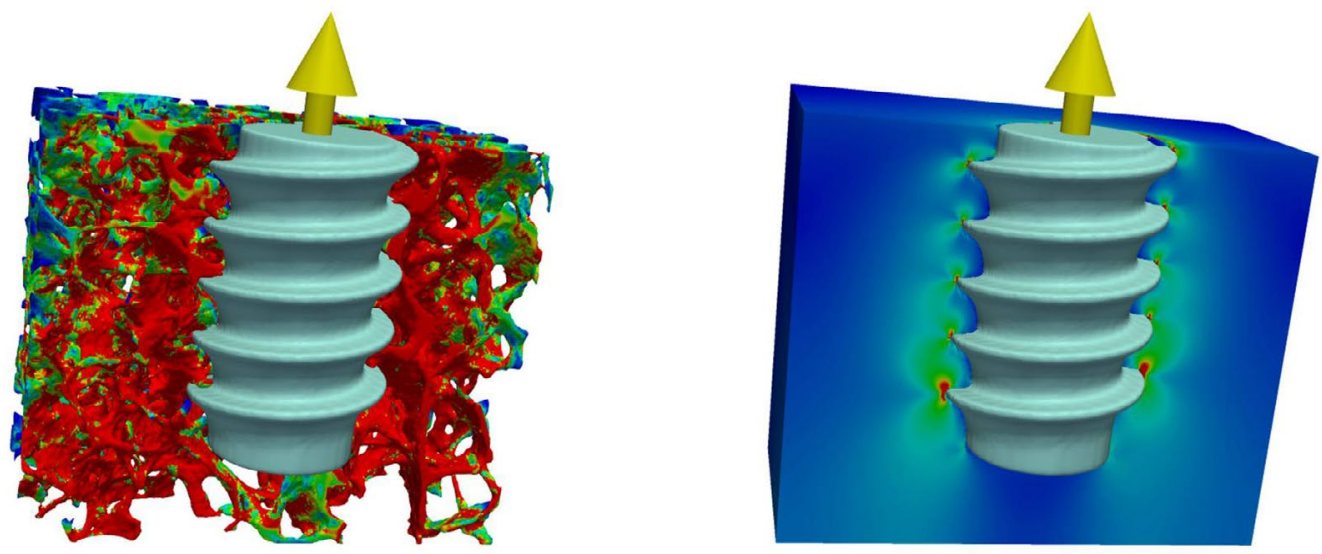

Von Mises Stress [MPa]
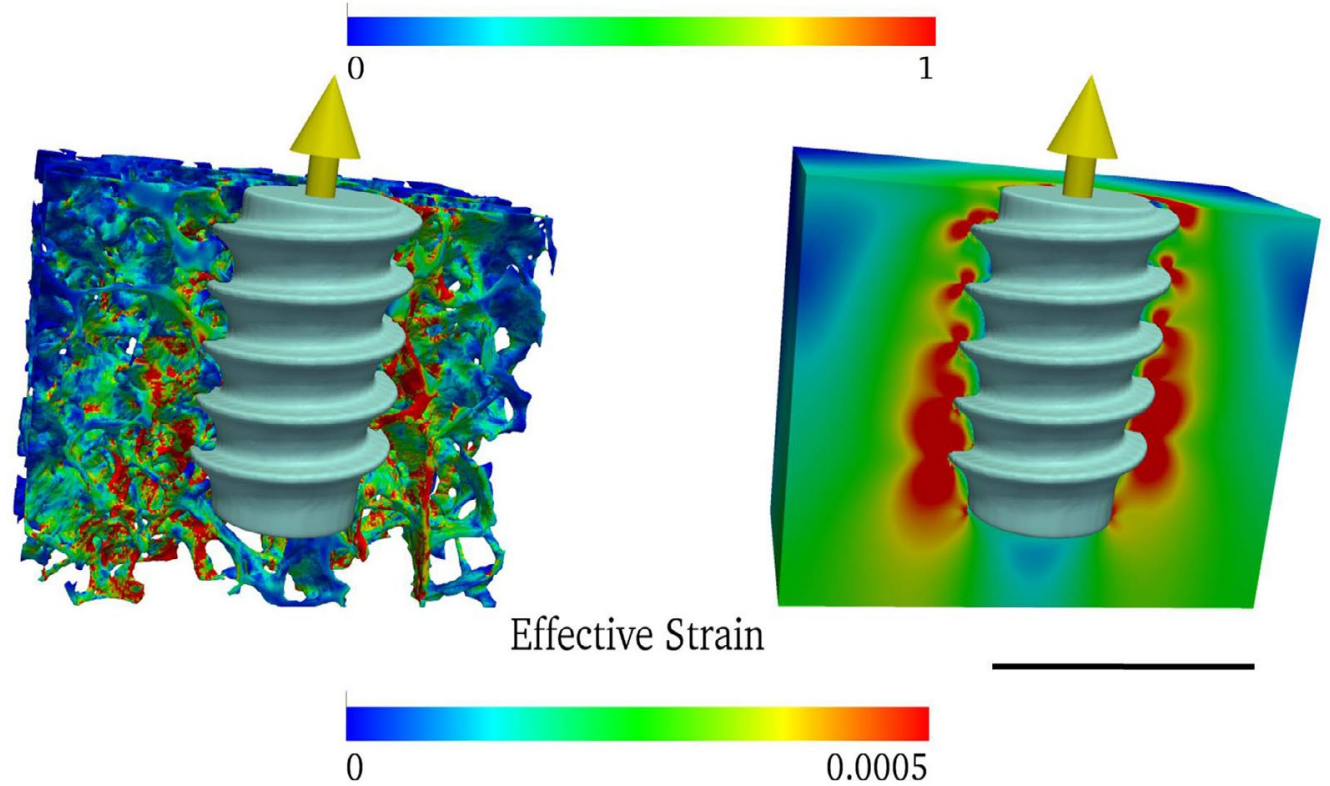

Fig. 3. Simulated distribution of von Mises stress (top) and effective strain (bottom) around a loaded screw in a discrete trabecular network (left) and in a continuum representation (right). The two bone samples have the same apparent Young's modulus and the screws are loaded with equal forces. Scale line $5 \mathrm{~mm}$.

fabric should be defined for trabecular bone (Cowin, 1991; Luo and An, 1998). Additionally, it is also unclear as to what should be the size of the sub-regions to get a proper measure of fabric using appropriate continuum assumptions, in order to get a correct mechanical behavior of the entire bone (Lenaerts and Van Lenthe, 2009). All this makes the modeling of bone's material properties in a continuum representation challenging. As discussed in the previous section, modeling trabecular bone with discrete trabeculae circumvents this problem, although it comes with the downside of having very large models. Fortunately, the last years have seen a strong increase in computational power such that these highly detailed models can now be solved. Such computer simulations allow investigating to what extent trabecular microarchitecture can be simplified in the context of continuum mechanics. Fig. 3 shows a set of continuum and discrete models of a screw in trabecular bone (Wirth et al., 2010). In order to solve these models, bone's Young's modulus is needed. The Young's modulus is a measure of the stiffness of a material, used to describe a material's behavior under mechanical load. It is often used together with the so-called Poisson ratio, which quantifies a material's deformation perpendicular to the direction of an applied outer load. These parameters belong to a simple and widespread linear material model which turns out to be accurate enough for many finite element models. For more complex material behavior and load cases, non-linear models have to be used. For the isotropic continuum models shown in Fig. 3, the apparent Young's modulus was determined in a separate FEA. Subsequently screws were inserted into the continuum models as well as in the original discrete computer models and boundary conditions that represented a pull-out test with the same force were applied. The obvious differences in calculated stresses and strain demonstrate the high potential of the discrete models to enhance our understanding of the peri-implant architecture on bone-implant mechanics.

Cortical bone displays transversely isotropic material behavior (Reilly and Burstein, 1975), which is related to the orientation of the osteons. Transversely isotropic materials are isotropic in all direction within one spatial plane, but are different along an axis perpendicular to this plane. As shown by Petrtyl et al. (Hert et al., 1994; Petrtyl et al., 1996), osteons typically align in the direction of the 
principal stresses. They found differences of more than $30 \%$ between the Young's moduli of the primary axis and the plane perpendicular to it, as well as significant differences in the related Poisson's ratios. Nevertheless, anisotropy of cortical bone was mainly neglected in most published studies on small endosseous implants (Genna et al., 2003; Huang et al., 2008; Kitamura et al., 2004; Motoyoshi et al., 2009; Veziroglu et al., 2008). In order to determine the effect of isotropic modeling of cortical bone, Lovald et al. (Lovald et al., 2006) compared FEA with isotropic and orthotropic material properties. They reported that the differences in peak Von Mises stress, a frequently used scalar value for identifying the local stress state, in two mandibular models were within $10 \%$ and therefore decided for isotropic material behavior. Only a few studies decided for transversely isotropic material properties, such as Huang et al. (Huang et al., 2005). Natali et al. (Natali et al., 2008) used an orthotropic modeling of cortical bone in the mandible and concluded that modeling anisotropy yields more appropriate results than assuming isotropy. Furthermore, the authors pointed out the need for more experimental data on the anisotropy of bone, as a few related questions are still open. In another study (Natali and Pavan, 2002), the same authors also tried to estimate the anisotropic field in the lamina dura around a dental implant by applying the findings of Petrtyl et al. (Hert et al., 1994; Petrtyl et al., 1996). Therefore, they made a separate FEA in which they determined the principle stress directions that then were chosen as axes of orthotropy. Based on this calculation, they used two different local element coordinate systems perpendicular to the screw surface; nevertheless, it was not reported what impact this procedure had on the final results.

The most straightforward approach to simulate changes in materials for FEA is varying their fundamental material properties. Alonso-Vázquez et al. (Alonso-Vázquez et al., 2004a) decided on such an approach and reduced Young's modulus for cortical and trabecular bone in order to analyze the influence of low bone quality on implant stability. The same approach was chosen by Zampelis et al. (Zampelis et al., 2007). These studies gave insight into the effects of changes in material elasticity on the overall structural competence of the implant constructs and may be reasonable for analyses of non-local phenomena in bones. However, it is obvious that such simplified material models cannot account for all characteristics of low quality bone. From an engineering point of view it would especially be interesting to know whether these simplified material models would lead to correct results for the immediate bone-implant interface, as well as for peri-implant bone, which are highly localized phenomena. The majority of all studies on small endosseous implants assumed cortical and trabecular bone to be fully linear elastic. Again, this simplification was mostly a trade-off between the estimated information gain and the expected high additional computational costs needed for performing a nonlinear analysis (Genna et al., 2003). Another motivation was that if the mechanical competence of a larger structure was intended to be analyzed, local non-linear phenomena were assumed to be negligible (Cegoñino et al., 2004). Nevertheless models exist that use visco-elasto-plastic material behavior for bone. Natali et al.
(Natali et al., 2008) used such a model to analyze inelastic and time-dependent local phenomena.

In most publications, implant materials were made of approved well-investigated metal alloys, for which the material properties are well-known. The material properties were generally considered as being isotropic, homogeneous and linear elastic (Huang et al., 2008; Natali et al., 2008; Veziroglu et al., 2008); appropriate values were taken from the literature or were provided by manufacturers. As the elastic moduli of most implants are more than one magnitude higher than those of the surrounding bone, such simplifications can be considered justified. Including non-linear material characteristics is only necessary for implants that have a non-linear behavior in the same range as the adjacent biological tissues. As an example, Genna et al. (Genna et al., 2003) used a hyperelasic model by Storakers (Storakers, 1986) to integrate a soft material layer in a new kind of dental implant design mimicking the presence of the periodontal ligament.

Implants with coatings, multi-material implants and novel biodegradable materials can make the definition of proper material properties much more demanding. Regarding novel biomaterials, Maurer et al. (Maurer et al., 2002) used bioresorbable screws made from poly-L-lactic and polylactide copolymer. Therefore, they used material properties given by the manufacturers but did not declare explicitly what kind of material model they decided on. For other prototype bioresorbable screws and plates, Rikli et al. (Rikli et al., 2002) determined tensile strength as well as Young's modulus and Poisson's ratio by mechanical testing. Although they did not state it clearly, the presented material properties let one assume that they used a linear elastic isotropic material model similar to Maurer et al., which seemed to be reasonable as they reported good correspondence of their FEA results with experimental biomechanical tests.

\section{Loading characteristics}

As a third critical component for FEA of bone-implant constructs, the loads acting on the construct have to be represented accurately. For conceptual models with simple geometries, the load and boundary conditions can be applied relatively easily (Zhang et al., 2004). This is often the case for many two-dimensional (2D) models, such as presented by Goldhahn et al. (Goldhahn et al., 2005), which allow analyzing some kinds of problems in a fast but still accurate way. Nevertheless, models in 2D require certain presumptions, for example planar or axial symmetric strain/stress fields, which can hardly be found in real bones. Furthermore, the limited spatial size of 2D models often makes it impossible to apply known boundary conditions. This is especially important as 2D models are highly sensitive to the choice of boundary conditions (Geng et al., 2004).

Loading conditions need to be known in more detail for three-dimensional models. However, only in few cases in vivo forces have been measured directly, such as for the hip (Bergmann et al., 1993), knee (Heinlein et al., 2009), shoulder (Westerhoff et al., 2009) and jaw (Morneburg and Proschel, 2002). Further complicating the load estimates is that physiological loads and boundary conditions are 
patient-specific and depend on body height, body weight, age and level of activity.

Local in situ load conditions are in most cases not precisely known (Van Lenthe and Müller, 2006). In these cases, it is beneficial to model entire bones for which the natural boundary conditions can be estimated much better. The locations of the attachments of the tendons and ligaments, as well as their orientations and related muscle forces usually are known or can be estimated, and therefore easily be integrated in FE models. Another advantage of this approach is that such bigger models are less sensitive to the way in which load is applied. For these models, St. Venant's principle states that the stress state at locations with sufficient distance from the load application areas are only affected by the overall magnitude and direction of the forces, and not by the specific details of the load application. This approach was often followed for models of bone-implant constructs (Lovald et al., 2006). It was recommended for dental implants by Van Staden et al. (Van Staden et al., 2006) who proposed to evaluate the entire jawbone for its contribution to the force exerted on a dental implant, a guideline that surely also holds true for orthopedic implants. Furthermore, following these principles also allows the analysis of multiple components, whereas the analytical determination of the load transfer to a specific component of such a construct is not possible. Such models can also include multiple bones, as was presented by Alonso-Vázquez et al. (Alonso-Vázquez et al., 2004a), who applied a loading case which simulated external torque and dorsiflexion on a model of a tibia and talus, in order to determine the stability of different kinds of implant configurations for ankle arthrodesis. The contact between the tibia and the talus was modeled frictionless, which is a reasonable assumption for synovial joints where friction is very low (Stachowiak et al., 1994). Nevertheless, friction is still present. Unfortunately, including friction in order to have more accurate contact conditions was not feasible because of numerical problems (Alonso-Vázquez et al., 2004b).

\section{Bone - implant interaction}

One of the greatest challenges in FE modeling of implant behavior is the representation of the bone-implant interface. Unfortunately, the mechanisms that occur at this interface are still largely unknown. In order to describe different interface behavior for dental implants Natali et al. (Natali et al., 2008) specified three basic interface models that could also be applied to small endosseous implants:

1. The first model assumes a "perfect continuity" between implant and bone; hence, no detachment phenomena are present.

2. In the second model, bone and implant are perfectly detached without cohesive forces between bone and implant, so that only sliding is possible. This model can be expanded by adding frictional effects, such as proposed by Huang et al. (Huang et al., 2008). 3. The third model includes cohesive contact between bone and implant but allows detaching when interfacial stresses reach a certain level.

The first interface model is the most commonly applied model for FEA of endosseous implants (Baggi et al., 2008;
Genna et al., 2003; Koca et al., 2005; Veziroglu et al., 2008). It allows circumventing additional computational costs that usually arise from more complex boundary conditions. For a model including multiple implants and bones, Alonso-Vazquez (Alonso-Vázquez et al., 2004a) simulated a partial thread on endosseous implants by only applying a partial fully bonded connection between implant and bone. Nevertheless, this first model must be regarded as an idealized state of perfect osseointegration, which for dental implants is usually present 4-6 months after the implantation ((Brånemark et al., 1977) as cited by Huang et al. (Huang et al., 2008)) but may never occur in osteoporotic bone.

The second interface model assumes that mechanical interlocking on the macroscopic scale dominates over the effects on the microscopic scale, such as friction. This scenario represents primary implant stability. This interface model was for example used by Hansson and Werke (Hansson and Werke, 2003). Huang et al. (Huang et al., 2008) added friction to this interface model. They analyzed different kinds of models for the bone-implant interface in order to investigate the effects of immediate loading of dental implants. To each implant they applied four different boundary conditions, ranging from low friction to total bonding. The authors proposed that the different friction coefficients were related to different surface roughness of the implant. The interfaces were simulated using non-linear surface contact elements. When increasing the friction coefficients they found a decrease of peri-implant peak von Mises stresses, with perfect osseointegration leading to lowest peak stresses. Furthermore perfect osseointegration gave lowest compressive principal stresses in peri-implant bone. These analyses demonstrate the importance of an accurate knowledge of the real frictional behavior at the interface.

The third interface model represents a case of more realistic osseointegration, where non-perfect mechanical interlocking also occurs on the micro level; nevertheless, critical model parameters can only be estimated. It is known that the interface strength depends on many factors, including implant surface characteristics, and can even be patient specific (Brunski, 1999; Li et al., 1997). Hence it is not surprising that a wide range of interface strength values have been proposed in the literature. The influence of different parameters can be seen in a publication by Natali et al. (Natali et al., 2008) who analyzed critical shear and tensile stresses, and compared the mechanical loading resulting from such interfaces with the two other models. Based on their findings, Natali et al. proposed using the fully-connected interface as a reference to evaluate maximum effects on stress and plastic deformation, as this kind of interface turned out to be the most critical for the implant construct they analyzed. As this disagrees with the findings of Huang et al. (Huang et al., 2008), one must presume that the influence of the contact conditions is also model-dependent.

\section{Modeling of bone-implant failure}

Where and how a small endosseous implant will fail may depend on local bone mechanical properties. A proper analysis of the critical bone qualities is hampered by the fact 
that these properties are not constant around an implant. Furthermore, if a certain volume around the implant is of importance, it is not known what bone volume is critical for proper implant fixation. In order to assess if the numerically simulated load cases would cause failure in reality, so called failure criteria have to be implemented. They typically analyze a combination of stresses or strains at a certain position or a region and relate them to a critical threshold value or state. During the last decades a multitude of stress-based (Fenech and Keaveny, 1999; Keyak and Rossi, 2000; Pietruszczak et al., 1999; Zysset and Rincon, 2006) and strain-based (Bayraktar et al., 2004; Lotz et al., 1991) failure criteria for bone have been proposed. For an overview and discussion of these different failure criteria we refer the reader to the review by Doblaré et al. (Doblaré et al., 2004). As discussed by Doblaré et al., there is still no common agreement upon the theoretical foundation for such a criterion, as the actual mechanisms behind it are still mostly unknown. The authors also pointed out that the discrepancies between the different criteria also originated from the fact that cortical and trabecular bone were not differentiated in the models and also anisotropy was mostly neglected. Doblaré et al. therefore concluded that more research has to be done in order to set up a common bone failure theory.

Nevertheless, for bone-implant constructs, von Mises stress is by far the most regularly analyzed parameter in bone (Lovald et al., 2006). For models of bone-implant constructs Natali et al. (Natali and Pavan, 2002) compared Tsai-Wu and von Mises stress criterion and found that von Mises was less conservative than Tsai-Wu. They furthermore mentioned that for their model the isotropic von Mises criterion gave a non-realistic description of the bone failure around the implant under load. Despite the work that has been done it seems that more experimental data on bone anisotropy and strength are necessary to obtain proper failure criteria (Fenech and Keaveny, 1999; Keaveny et al., 1999; Natali and Pavan, 2002).

Further complicating the definition of a failure criterion for bone-implant constructs is the fact that failure does not necessarily occur in the bone tissue. Failure can also arise inside the implant and at the bone-implant interface. For implants made of conventional materials, established failure criteria from mechanical engineering can be used for implant strength assessment; among these are maximum von Mises stress for ductile titanium alloys (Lovald et al., 2006) and principal stresses for brittle materials, like dental ceramics (Korkmaz, 2007). How failure occurs at the interface surely depends much on the quality and quantity of bone and tissue ingrowth, which is affected by many different factors. However, since the experimental and theoretical knowledge about bone-implant debonding is even more limited than that of failure inside the bone tissue, more research is certainly needed before final conclusions can be drawn.

\section{Does Time Matter? - Bone as a Living Structure}

In an ideal scenario, an implant will be well-fixed in the bone, stay at the same location and fulfill its task over its entire lifetime. However, since it is a living tissue, bones are subject to constant remodeling of their architecture. This ability to remodel allows them to adapt to new conditions (Frost, 1990; Wolf, 1892) such as changes in loading due to growth. The placement of an implant will lead to changes in the mechanical environment, and can lead to local bone adaptation. Furthermore, bone-implant interaction may lead to an intimate bond between them; hence interface conditions can change over time. Consequently, knowledge on the current bone status is not necessarily enough to predict implant stability over time. To emphasize this time-dependent bone behavior, a distinction is generally made between primary (short-term) and secondary (midand long-term) implant stability.

Primary implant stability has always been central in orthopedic applications; it may also become even more important in dental medicine due to new treatment schemes that include initial loading of implants (Huang et al., 2008). For a thorough analysis of long-term stability, bone remodeling has to be taken into account. Such analyses may lead to the discovery of conflicting design criteria: thread geometry optimal for primary implant stability does not necessarily have to be optimal for osseointegration, and vice versa. Therefore, accounting for the expected occurrence of bone adaptation could help control implant stability over time. Whereas in dental medicine an increase of bone mass is required to ensure long-term implant stability, in many orthopedic applications, only mid-term stability with a constant bone mass or just a slight increase is desirable in order to be able to remove implants after completed fracture healing (Ilchmann and Parsch, 2006; Jago and Hindley, 1998; Warner et al., 1994). In orthopedic trauma management, good long-term stability can also be important, especially in cases where the implants are not intended to be explanted again. Such applications are common for patients with low bone quality, such as presented by Goldhahn et al. (Goldhahn et al., 2006) who optimized an implant design for osteoporotic bone resulting in increased peri-implant bone volume when implanted into an ovine model of osteoporosis.

Several FEA-based models have been presented for bone remodeling. A first such model including an implant was presented in 1987 by Huiskes et al. (Huiskes et al., 1987) who focused on endoprostheses. Since then many similar concepts for bone remodeling have been developed, whereby a few have been applied to small endosseous implants (Tsubota et al., 2003). A relatively new concept for bone remodeling around small endosseous implants, using continuum trabecular bone was presented by $\mathrm{Li}$ et al. (Li et al., 2007). In contrast to other models, this model also takes into account resorption due to overloading, which often occurs in dental implants. Cegoñino et al. (Cegoñino et al., 2004) presented a remodeling algorithm which they applied on a model of a complete fracture fixation of the distal femur using endosseous implants. They found interesting resorption phenomena but also stated that the accuracy of the predicted remodeling was quite restricted due to several simplifications including the missing microstructure. Such microstructures are present in a model with a small endosseous implant that was presented by Tsubota et al. (Fig. 4) (Tsubota et al., 2003). In this model local 
nonuniformity of equivalent stress was hypothesized to trigger remodeling. By this, they could show that trabecular bone adapted to the new structure around the implant - something that was expected but has never been shown for a vertebra. Although they stated that this model had potential for the prediction of long-term implant fixation, such models have yet to be presented.

\section{Accuracy of Finite Element Models}

FEA is a mathematically proven technology. Its use in the analyses of bones and bone samples has become widely accepted. It is a validated technique (Van Rietbergen, 2001) to calculate stresses and strains in complex bone microstructures (Van Rietbergen et al., 1995) as well as to calculate apparent stiffness and modulus (Chevalier et al., 2007; Van Rietbergen et al., 1997).

The accuracy of FEA on bone-implant constructs can be determined by comparing its outcomes relative to experimental tests. However, the number of studies that performed such validations is low (Geng et al., 2004; Huang et al., 2005; Lovald et al., 2006; Rikli et al., 2002). For that purpose synthetic bones with known material parameters are often used; the use of synthetic bones helps to eliminate uncertainties about material properties in the FE model. Huang et al. used an experimental model with strain gauges that were oriented in the direction of minimal principal strain. They found relatively large differences in experimentally measured and simulated strain between $10 \%$ and $50 \%$, depending on the loading magnitude. Nevertheless the authors reported a consistent relationship between the results from FEA and biomechanical tests, leading to a very high correlation $\left(r^{2}=0.97\right)$ between them. Therefore, although not giving correct results in absolute numbers, FEA was a good predictor for the behavior of one model relative to another one. Rikli et al. compared the forces and displacements determined by FEA with real biomechanical experiments on a synthetic bone and found a good correspondence of the results regarding loads and load distribution. Another approach was chosen by Lovald et al. who used a validated model of the mandibula to determine the accuracy of a new model by ascertaining that similar loads in both models produced similar results.

There is quite a number of potential error sources involved in the generation of an FEA of bone-implant constructs. Ideally, the sensitivity of FE-models to different parameters, such as changes in geometry and material properties, should be tested. Such sensitivity and uncertainty analyses are an important part of quality control in for example aerospace and car industry. Although in several studies the effects of changes in bone material properties or boundary conditions have been studied, for example by Geng et al., to the best of our knowledge there is no report on a more systematic sensitivity analysis, for example by using a factorial analysis. Furthermore, no probability studies have been performed in order to include uncertainties resulting from material and manufacturing tolerances. For the implant side this is reasonable because uncertainties in the dimensions and material properties of the implants are very low compared to that of bone.

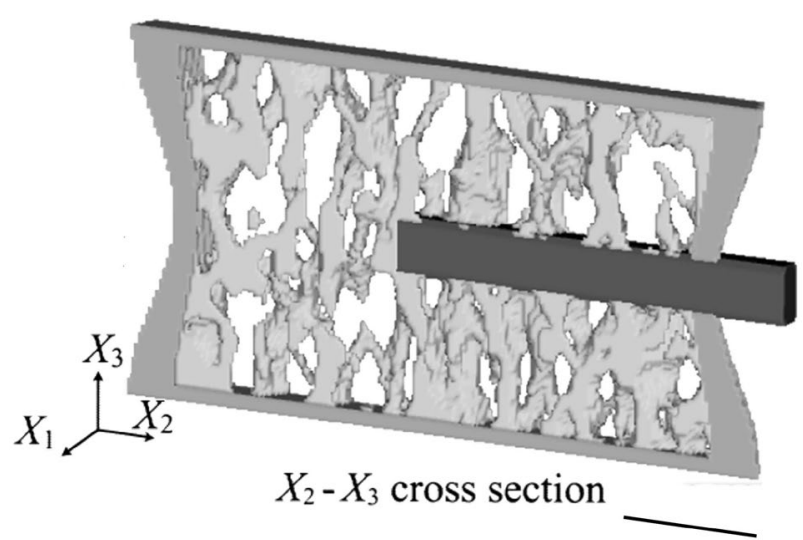

Fig. 4. Cross-section of a micro-finite element model of a vertebral body with a fixation screw. (From: Tsubota et al. (2003) with kind permission from Springer Science and Business Media). Scale line $10 \mathrm{~mm}$.

\section{Concluding Remarks}

In this paper we have presented a review on finite element analysis (FEA) of bone-implant constructs, and have listed the necessary prerequisites. We demonstrated that FEA has become a widely used tool for the assessment of the behavior of small endosseous implants in bone. FEA has led to seemingly reasonable outcomes; yet in many cases it is not clear how accurate they are. A main reason for this is that generally the models include several simplifications for material behavior and contact conditions, which up to now have mostly not been validated by experimental measurements. Future experiments that test the predictive capacity of FEA should preferably be conducted on a specimen-specific basis because of the large specimen-specific variations in bone micro-architecture. Hence, all applied techniques should ideally work non-destructively to allow experimental biomechanical measurements on the same specimens for validation purposes.

Most osteoporotic fractures occur in regions with a high content of trabecular bone and therefore the relatively complex material properties of bone have to be modeled adequately. However, bone porosity and the resulting heterogeneity and anisotropy of trabecular bone have been mostly neglected up to now; instead, trabecular bone has been modeled as a semi-continuum. This is somewhat surprising as in many cases geometrical parameters of the implants, such as diameter and pitch are in the range of the bone pore sizes (Fig. 5). A more realistic model for trabecular bone, which also includes anisotropy and heterogeneity, is required. Especially in osteoporosis, which is characterized by a reduction in bone volume, trabecular architecture is even further away from the assumed homogeneity in current continuum models, further emphasizing the need for models that explicitly represent the intricate trabecular microarchitecture. This may lead to further insight into the effects of thread design, which is important for load transfer to peri-implant bone by interlocking $a b$ inito. 
The failure mechanisms in bone-implant constructs are still largely unknown. Established failure criteria from mechanical engineering do not hold true for such a complex material as bone. Out of the many different kinds of bone failure criteria that exist, only few have been used for FEA of small endosseous implants. Up to now, none of these criteria has really been established as a gold standard. We therefore believe that more work on failure criteria of bone, trabecular bone in particular, must be performed. In order to validate this work, novel experimental techniques may be needed in which local trabecular bone deformation and failure can be monitored over time.

Due to the lack of precision in current FE approaches to model bone-implant systems, the application in in vivo situations seems somewhat premature. Nevertheless, high-resolution CT might make it possible to image the precise implant location and its peri-implant bone micro-architecture in patients. Furthermore, automated meshing techniques are already available (Viceconti et al., 2004). Together with new high performance FE solvers this may allow patient-specific FEA. Hence, the potential to use these techniques in a clinical environment is certainly present. Nevertheless, artifacts induced by most materials used for implants, still do not allow measuring the bone-implant contact surface, which would provide important information to assess implant stability. Up to now, measurements can only be done for small implants, made out of certain alloys and using ex vivo CT-systems with relatively high radiation doses. We do not expect that such measurements will be possible in vivo in the near future.

The prediction of long-term stability of implants is even more challenging because the bone may adapt to the modified local mechanical environment but also to hormonal factors. For this reason a proper knowledge of the effects that diseases like osteoporosis have on bone architecture may be very beneficial in order to be able to model the (per definitionem) specimen-specific differences in bone quality.

In conclusion, the literature has already shown that FEA can lead to insight into implant stability. We believe that the understanding of implant stability can be enhanced by using FE-models where the intricate trabecular architecture is incorporated. Validation of these highly detailed models is crucial and can be performed by comparing FEA results to experimental measurements of implant stiffness and strength. Image-based failure analysis may be very beneficial in further elucidating the still largely unknown failure mechanisms in the peri-implant bone. FEA of the image-based failure analyses seem indispensible for a precise quantification of the failure mechanisms. Such microstructural analyses are also needed for the proper quantification of the bone-implant interface characteristics. Another novel field of application for FEA could be screw augmentation with bone cement. Here FEA could help to analyze how the presence of cement is affecting load transfer, which could help to optimize the amount of bone cement used. We hypothesize that this new knowledge will be valuable for the development of new implant designs, especially for use in osteoporotic bone.

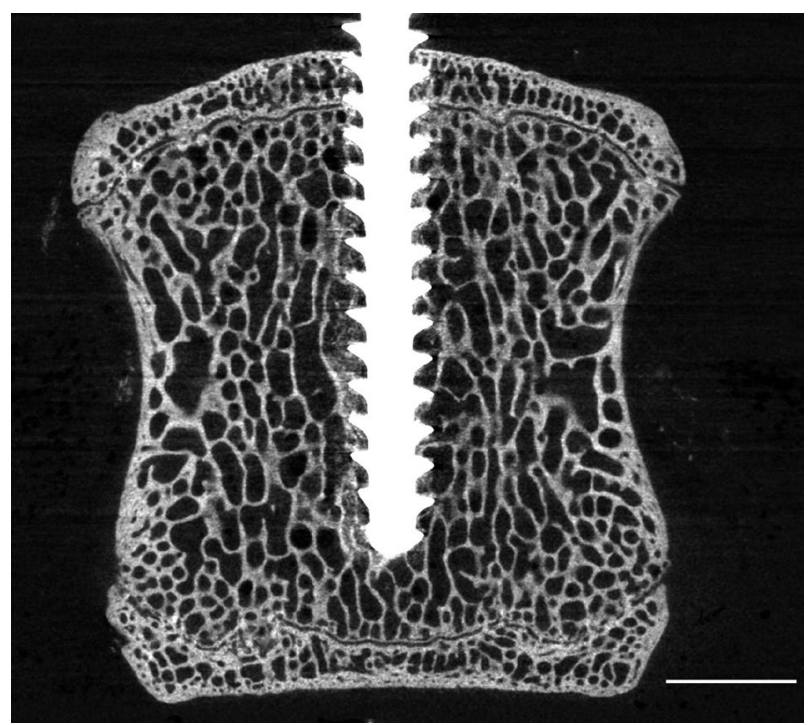

Fig. 5. MicroCT image of a small endosseous implant commonly used in spine surgery (Synthes StarLock system) implanted in an ovine vertebral body. Scale line $5 \mathrm{~mm}$.

\section{Acknowledgments}

The authors acknowledge the support of AO Foundation (network grant CPP1).

\section{References}

Alonso-Vázquez A, Lauge-Pedersen H, Lidgren L, Taylor M (2004a) The effect of bone quality on the stability of ankle arthrodesis. A finite element study. Foot Ankle Int 25: $840-850$.

Alonso-Vázquez A, Lauge-Pedersen H, Lidgren L, Taylor M (2004b) Initial stability of ankle arthrodesis with three-screw fixation. A finite element analysis. Clin Biomech 19: 751-759.

Arbenz P, Van Lenthe GH, Mennel U, Muller R, Sala M (2007) Multi-level mu-finite element analysis for human bone structures. Applied Parallel Computing 4699: 240250 .

Arens D, Sigrist I, Alini M, Schawalder P, Schneider E, Egermann M (2007) Seasonal changes in bone metabolism in sheep. Vet J 174: 585-591.

Baggi L, Cappelloni I, Di Girolamo M, Maceri F, Vairo $\mathrm{G}$ (2008) The influence of implant diameter and length on stressdistribution of osseointegrated implants related to crestal bone geometry: A three-dimensional finite element analysis. J Prosthet Dent 100: 422-431.

Bayraktar HH, Gupta A, Kwon RY, Papadopoulos P, Keaveny TM (2004) The modified super-ellipsoid yield criterion for human trabecular bone. J Biomech Eng 126: 677-684.

Beck TJ, Ruff CB, Bissessur K (1993) Age-related changes in female femoral neck geometry: Implications for bone strength. Calcif Tissue Int 53 Suppl 1: S41-46.

Bergmann G, Graichen F, Rohlmann A (1993) Hip joint loading during walking and running, measured in two patients. J Biomech 26: 969-990. 
Bonnaire F, Zenker H, Lill C, Weber AT, Linke B (2005) Treatment strategies for proximal femur fractures in osteoporotic patients. Osteoporos Int 16 Suppl 2: S93-S102.

Boonen S, Dejaeger E, Vanderschueren D, Venken K, Bogaerts A, Verschueren S, Milisen K (2008) Osteoporosis and osteoporotic fracture occurrence and prevention in the elderly: A geriatric perspective. Best Pract Res Clin Endocrinol Metab 22: 765-785.

Brånemark PI, Adell R, Breine U, Hansson BO, Lindstrom J, Ohlsson A (1969) Intra-osseous anchorage of dental prostheses. I. Experimental studies. Scand J Plast Reconstr Surg 3:81-100.

Brånemark $\mathrm{PI}$, Hansson $\mathrm{BO}$, Adell R, Breine U, Lindstrom J, Hallen O, Ohman A (1977) Osseointegrated implants in the treatment of the edentulous jaw. Experience from a 10-year period. Scand J Plast Reconstr Surg Suppl 16: 1-132.

Brunski JB (1999) In vivo bone response to biomechanical loading at the bone/dental-implant interface. Adv Dent Res 13: 99-119.

Burge R, Dawson-Hughes B, Solomon DH, Wong JB, King A, Tosteson A (2007) Incidence and economic burden of osteoporosis-related fractures in the United States, 2005-2025. J Bone Miner Res 22: 465-475.

Cegoñino J, Garcia Aznar JM, Doblare M, Palanca D, Seral B, Seral F (2004) A Comparative analysis of different treatments for distal femur fractures using the finite element Method. Comput Methods Biomech Biomed Engin 7: 245-256.

Chen WP, Tai CL, Shih CH, Hsieh PH, Leou MC, Lee MS (2004) Selection of fixation devices in proximal femur rotational osteotomy: Clinical complications and finite element analysis. Clin Biomech 19: 255-262.

Chevalier Y, Pahr D, Allmer H, Charlebois M, Zysset P (2007) Validation of a voxel-based FE method for prediction of the uniaxial apparent modulus of human trabecular bone using macroscopic mechanical tests and nanoindentation. J Biomech 40: 3333-3340.

Chrischilles EA, Butler CD, Davis CS, Wallace RB (1991) A model of lifetime osteoporosis impact. Arch Intern Med 151:2026-2032.

Consensus Development Conference (1993) Diagnosis, prophylaxis, and treatment of osteoporosis. Am J Med 94: 646-650.

Cowin SC (1985) The relationship between the elasticity tensor and the fabric fensor. Mech Mater 4: 11.

Cowin SC (1991) The mechanical properties of cancellous bone. CRC Press, Boca Raton, FL.

Doblaré M, Garcia JM, Gomez MJ (2004) Modelling bone tissue fracture and healing: A review. Engg Fracture Mech 71: 1809-1840.

Egermann M, Goldhahn J, Holz R, Schneider E, Lill CA (2008) A sheep model for fracture treatment in osteoporosis: Benefits of the model versus animal welfare. Lab Anim 42:453-464.

Fenech CM, Keaveny TM (1999) A cellular solid criterion for predicting the axial-shear failure properties of bovine trabecular bone. J Biomech Eng 121: 414-422.

Firoozbakhsh K, Decoster TA, Moneim MS (1994) Bone screw design - Analytical formulation. Proc 16th Ann Internat Conf IEEE Engg Med Biol Soc - Engineering
Advances: New Opportunities for Biomedical Engineers, Pts 1 and 2, pp. 283-284.

Frankel BM, Jones T, Wang C (2007) Segmental polymethylmethacrylate-augmented pedicle screw fixation in patients with bone softening caused by osteoporosis and metastatic tumor involvement: A clinical evaluation. Neurosurgery 61: 531-537.

Frost HM (1990) Skeletal structural adaptations to mechanical usage (Satmu): 1. Redefining Wolff's law: The bone modeling problem. Anat Rec 226: 403-413.

Gabet Y, Muller R, Levy J, Dimarchi R, Chorev M, Bab I, Kohavi D (2006) Parathyroid hormone 1-34 enhances titanium implant anchorage in low-density trabecular bone: A correlative micro-computed tomographic and biomechanical analysis. Bone 39: 276-282.

Geng JP, Ma QS, Xu W, Tan KB, Liu GR (2004) Finite element analysis of four thread-form configurations in a stepped screw implant. J Oral Rehabil 31: 233-239.

Genna F, Paganelli C, Salgarello S, Sapelli P (2003) Mechanical response of bone under short-term loading of a dental implant with an internal layer simulating the nonlinear behaviour of the periodontal ligament. Comput Methods Biomech Biomed Engin 6: 305-318.

Goldhahn J, Seebeck J, Frei R, Frenz B, Antoniadis I, Schneider E (2005) New implant designs for fracture fixation in osteoporotic bone. Osteoporos Int 16 Suppl 2: S112-119.

Goldhahn J, Neuhoff D, Schaeren S, Steiner B, Linke B, Aebi M, Schneider E (2006) Osseointegration of hollow cylinder based spinal implants in normal and osteoporotic vertebrae: A sheep study. Arch Orthop Trauma Surg 126: 554-561.

Gomez-Benito MJ, Garcia-Aznar JM, Doblare M (2005) Finite element prediction of proximal femoral fracture patterns under different loads. J Biomech Eng 127: 9-14.

Gupta KK, Meek JL (1996) A brief history of the beginning of the finite element method. Int J Numer Methods in Eng 39: 3761-3774.

Hansson S, Werke M (2003) The implant thread as a retention element in cortical bone: The effect of thread size and thread profile: A finite element study. J Biomech 36: 1247-1258.

Heinlein B, Kutzner I, Graichen F, Bender A, Rohlmann A, Halder AM, Beier A, Bergmann G (2009) ESB Clinical Biomechanics Award 2008: Complete data of total knee replacement loading for level walking and stair climbing measured in vivo with a follow-up of 6-10 Months. Clin Biomech 24: 315-326.

Hert J, Fiala P, Petrtyl M (1994) Osteon orientation of the diaphysis of the long bones in man. Bone 15: 269-277.

Huang HL, Huang JS, Ko CC, Hsu JT, Chang CH, Chen MY (2005) Effects of splinted prosthesis supported a wide implant or two implants: A three-dimensional finite element analysis. Clin Oral Implants Res 16: 466-472.

Huang HL, Hsu JT, Fuh LJ, Tu MG, Ko CC, Shen YW (2008) Bone stress and interfacial sliding analysis of implant designs on an immediately loaded maxillary implant: A non-linear finite element study. J Dent 36: 409-417.

Huiskes R, Weinans H, Grootenboer HJ, Dalstra M, Fudala B, Slooff TJ (1987) Adaptive bone-remodeling 
theory applied to prosthetic-design Analysis. J Biomech 20: $1135-1150$.

Ilchmann T, Parsch K (2006) Complications at screw removal in slipped capital femoral epiphysis treated by cannulated titanium screws. Arch Orthop Trauma Surg 126: $359-363$.

Jago ER, Hindley CJ (1998) The removal of metalwork in children. Injury 29: 439-441.

Kanis JA, Johnell O (2005) Requirements for DXA for the management of osteoporosis in Europe. Osteoporos Int 16: 229-238.

Keaveny TM, Wachtel EF, Zadesky SP, Arramon YP (1999) Application of the Tsai-Wu quadratic multiaxial failure criterion to bovine trabecular bone. J Biomech Eng 121: 99-107.

Kesson CM, Morris N, Mc CA (1947) Generalized osteoporosis in old age. Ann Rheum Dis 6: 146-161.

Keyak JH, Rossi SA (2000) Prediction of femoral fracture load using finite element models: An examination of stress- and strain-based failure theories. J Biomech 33: 209-214.

Kitamura E, Stegaroiu R, Nomura S, Miyakawa O (2004) Biomechanical aspects of marginal bone resorption around osseointegrated implants: Considerations based on a three-dimensional finite element analysis. Clin Oral Implants Res 15: 401-412.

Koca OL, Eskitascioglu G, Usumez A (2005) Three-dimensional finite-element analysis of functional stresses in different bone locations produced by implants placed in the maxillary posterior region of the sinus floor. J Prosthet Dent 93: 38-44.

Koistinen A, Santavirta SS, Kroger H, Lappalainen R (2005) Effect of bone mineral density and amorphous diamond coatings on insertion torque of bone screws. Biomaterials 26: 5687-5694.

Korkmaz HH (2007) Evaluation of different miniplates in fixation of fractured human mandible with the finite element method. Oral Surg Oral Med Oral Pathol Oral Radiol Endod 103: e1-13.

Lang LA, Kang B, Wang RF, Lang BR (2003) Finite element analysis to determine implant preload. J Prosthet Dent 90: 539-546.

Lenaerts L, Van Lenthe GH (2009) Multi-level patient-specific modelling of the proximal femur. A promising tool to quantify the effect of osteoporosis treatment. Philos Transact A Math Phys Eng Sci 367: 2079-2093.

Lewitt RM, Bates RHT (1978) Image-reconstruction from projections .3. Projection completion methods (theory). Optik 50: 189-204.

Li J, Liao H, Fartash B, Hermansson L, Johnsson T (1997) Surface-dimpled commercially pure titanium implant and bone ingrowth. Biomaterials 18: 691-696.

Li J, Li H, Shi L, Fok AS, Ucer C, Devlin H, Horner K, Silikas N (2007) A mathematical model for simulating the bone remodeling process under mechanical stimulus. Dent Mater 23: 1073-1078.

Lotz JC, Cheal EJ, Hayes WC (1991) Fracture prediction for the proximal femur using finite element models: Part I - Linear analysis. J Biomech Eng 113: 353-360.

Lovald ST, Khraishi T, Wagner J, Baack B, Kelly J, Wood J (2006) Comparison of plate-screw systems used in mandibular fracture reduction: Finite element analysis. J Biomech Eng 128: 654-662.

Luo ZP, An KN (1998) A theoretical model to predict distribution of the fabric tensor and apparent density in cancellous bone. J Math Biol 36: 557-568.

Maurer P, Holweg S, Knoll WD, Schubert J (2002) Study by finite element method of the mechanical stress of selected biodegradable osteosynthesis screws in sagittal ramus osteotomy. Br J Oral Maxillofac Surg 40: 76-83.

Morgan EF, Bouxsein ML (2005) Use of finite element analysis to assess bone strength. BoneKEy-Osteovision 2: 12.

Morneburg TR, Proschel PA (2002) Measurement of masticatory forces and implant loads: A methodologic clinical study. Int J Prosthodont 15: 20-27.

Motoyoshi M, Inaba M, Ono A, Ueno S, Shimizu N (2009) The effect of cortical bone thickness on the stability of orthodontic mini-implants and on the stress distribution in surrounding bone. Int J Oral Maxillofac Surg 38: 13-18.

Müller R (2002) The Zurich experience: One decade of three-dimensional high-resolution computed tomography. Top Magn Reson Imaging 13: 307-322.

Müller R (2009) Hierarchical microimaging of bone structure and function. Nat Rev Rheumatol 5: 373-381.

Natali AN, Pavan PG (2002) A comparative analysis based on different strength criteria for evaluation of risk factor for dental implants. Comput Methods Biomech Biomed Engin 5: 127-133.

Natali AN, Carniel EL, Pavan PG (2008) Investigation of bone inelastic response in interaction phenomena with dental implants. Dent Mater 24: 561-569.

Patra AK, DePaolo JM, D’Souza KS, DeTolla D, Meenaghan MA (1998) Guidelines for analysis and redesign of dental implants. Implant Dent 7: 355-368.

Petrtyl M, Hert J, Fiala P (1996) Spatial organization of the Haversian bone in man. J Biomech 29: 161-169.

Pietruszczak S, Inglis D, Pande GN (1999) A fabric-dependent fracture criterion for bone. J Biomech 32: 1071-1079.

Ploeg H, Byrne N, García S, Kersh M, Nair D (2004) How accurate are solids models made from CT scan data? In: Proc 13th Bienn Conf Can Soc Biomech (CSB), Halifax, NS, Canada.

Ray NF, Chan JK, Thamer M, Melton LJ, 3rd (1997) Medical expenditures for the treatment of osteoporotic fractures in the United States in 1995: Report from the National Osteoporosis Foundation. J Bone Miner Res 12: 24-35.

Reilly DT, Burstein AH (1975) The elastic and ultimate properties of compact bone tissue. J Biomech 8: 393-405.

Rikli DA, Curtis R, Schilling C, Goldhahn J (2002) The Potential of bioresorbable plates and screws in distal radius fracture fixation. Injury 33 Suppl 2: B77-83.

Rybicki EF, Simonen FA, Weis EB, Jr (1972) On the mathematical analysis of stress in the human femur. J Biomech 5: 203-215.

Schneider E, Goldhahn J, Burckhardt P (2005) The challenge: Fracture treatment in osteoporotic bone. Osteoporos Int 16 Suppl 2: S1-2. 
Scott WA, Allum RL, Wright KW (1985) Implant-induced trabecular damage in cadaveric femoral necks. Acta Orthop Scand 56: 145-146.

Seebeck J, Goldhahn J, Stadele H, Messmer P, Morlock MM, Schneider E (2004) Effect of cortical thickness and cancellous bone density on the holding strength of internal fixator screws. J Orthop Res 22: 1237-1242.

Sowmianarayanan S, Chandrasekaran A, Kumar RK (2008) Finite element analysis of a subtrochanteric fractured femur with dynamic hip screw, dynamic condylar screw, and proximal femur nail implants - a comparative study. Proc Inst Mech Eng [H] 222: 117-127.

Stachowiak GW, Batchelor AW, Griffiths LJ (1994) Friction and wear changes in synovial joints. Wear 171: 135-142.

Storakers B (1986) On material representation and constitutive branching in finite compressible elasticity. J Mech Phys Solids 34: 125-145.

Tesk JA, Widera O (1973) Stress distribution in bone arising from loading on endosteal dental implants. J Biomed Mater Res 7: 251-261.

Tsubota K, Adachi T, Tomita Y (2003) Effects of a fixation screw on trabecular structural changes in a vertebral body predicted by remodeling simulation. Ann Biomed Eng 31: 733-740.

Tsumita M, Kokubo Y, Vult von Steyern P, Fukushima S (2008) Effect of framework shape on the fracture strength of implant-supported all-ceramic fixed partial dentures in the molar region. J Prosthodont 17: 274-285.

Van Lenthe GH, Müller R (2006) Prediction of failure load using micro-finite element Analysis models: Toward in vivo strength assessment. Drug Discovery Today: Technologies 3: 221-229.

Van Rietbergen B, Weinans H, Huiskes R, Odgaard A (1995) A new method to determine trabecular bone elastic properties and loading using micromechanical finite-element models. J Biomech 28: 69-81.

Van Rietbergen B, Kabel J, Odgaard A, Huiskes R (1997) Determination of trabecular bone tissue elastic properties by comparison of experimental and finite element results. In: Material Identification Using Mixed Numerical Experimental Methods (Sol H, Oomens C, eds), Kluwer, Dordrecht, the Netherlands, pp. 183-192.

Van Rietbergen B (2001) Micro-FE Analyses of Bone: State of the Art. Adv Exp Med Biol 496: 21-30.

Van Staden RC, Guan H, Loo YC (2006) Application of the finite element method in dental implant research. Comput Methods Biomech Biomed Engin 9: 257-270.

Veziroglu F, Uckan S, Ozden UA, Arman A (2008) Stability of zygomatic plate-screw orthodontic anchorage system: A finite element analysis. Angle Orthod 78: $902-$ 907.

Viceconti M, Davinelli M, Taddei F, Cappello A (2004) Automatic generation of accurate subject-specific bone finite element models to be used in clinical studies. J Biomech 37: 1597-1605.

Warner JG, Bramley D, Kay PR (1994) Failure of screw removal after fixation of slipped capital femoral epiphysis: The need for a specific screw design. J Bone Joint Surg Br 76: $844-845$.
Westerhoff P, Graichen F, Bender A, Halder A, Beier A, Rohlmann A, Bergmann G (2009) In vivo measurement of shoulder joint loads during activities of daily living. J Biomech 42: 1840-1849.

Williamson JF, Whiting BR, Benac J, Murphy RJ, Blaine GJ, O'Sullivan JA, Politte DG, Snyder DL (2002) Prospects for quantitative computed tomography imaging in the presence of foreign metal bodies using statistical image reconstruction. Med Phys 29: 2404-2418.

Wirth AJ, Müller R, van Lenthe GH (2010) Implant stability is affected by peri-implant bone microstructure In: 18th Ann Meet Eur Orthop Res Soc, Davos, Switzerland, in press.

Wolf J (1892) Das Gesetz der Transformation der Knochen. (The law on bone transformation), Springer-Verlag, Berlin, Germany.

Wolter D, Jürgens C (2006) Winkelstabile Verbindungen bei Osteosyntheseimplantaten. (Connections with angle stability in osteosynthesis implants). Trauma Berufskrankh 8: 206-211.

Zampelis A, Rangert B, Heijl L (2007) Tilting of splinted implants for improved prosthodontic support: A two-dimensional finite element analysis. J Prosthet Dent 97: S35-43.

Zarrinkalam MR, Beard H, Schultz CG, Moore RJ (2008) Validation of the sheep as a large animal model for the study of vertebral osteoporosis. Eur Spine J 18: 244-253.

Zhang QH, Tan SH, Chou SM (2004) Investigation of fixationscrew pull-out strength on human spine. J Biomech 37: 479-485.

Zysset P, Rincon L (2006) An alternative fabric-based yield and failure criterion for trabecular bone. In: Mechanics of Biological Tissues (Holzapfel GA, Ogden RW, eds), Springer Berlin Heidelberg, pp. 457-470.

\section{Discussion with Reviewers}

Reviewer I: Do the authors think that the osteoporotic bone situation in orthopedic districts can be compared with the situation in maxillary bones?

Authors: There seem to be obvious differences between these types of bones, such as differences in spatial dimensions and in the ratio between cortical and trabecular bone volume. Furthermore, even though osteoporosis is a systemic disease, bone quality and implant performance in the maxillary bones is not predicted well by bone loss at other skeletal sites (Tsolaki et al., 2009). Another potential difference may be that the quality of the cortical shell may be more important for dental implants than the quality of the trabecular bone whereas for many trauma implants the latter is crucial. What the sites have in common is that in patients suffering from osteoporosis, bone quality is impaired, special surgical techniques may be necessary and longer healing periods can be expected. Even more so, the basic implant anchoring mechanisms providing implant stability are expected to be identical. Hence, although these bones are different, failure mechanisms are expected to be 
similar and surgeons seem to be confronted with similar problems when they try to insert implants.

Reviewer II: What correlation do the authors expect between failure mechanisms in small versus large implants? Authors: Little data exists on bone microstructural deformation and fracture initiation in bone-implant constructs. Nonetheless we expect that the fundamental failure mechanisms do not depend on implant size, but presume that implant size could influence the relative importance of individual failure mechanisms in total implant performance. As an example, it seems reasonable that failure of a single trabecula, which has grown onto the implant, would affect anchorage of small implants more than it would affect the anchorage of larger ones, because it will play a more important role in load transfer for a small implant.

Reviewer II: How should novel experimental tests be designed, so that some of their limitations can be removed? Authors: If experimental tests will be compared with FEA models, it should be aimed to eliminate as many unknowns and potential error sources of the experimental tests as possible. Just to name three of them: First, all material properties of the involved components have to be known. Where they are not, they should be determined by separate measurements. For bone this would mean that inter- and intra-specimen differences in bone quality should be taken into account. Second, the inner and outer geometry of the components, including position and alignment have to be measured with high accuracy. Here, (high-resolution) CT-systems may be helpful. And third, the applied boundary conditions have to be well-defined. For example, simple bearings with unclear friction phenomena and improper embeddings in plastics or low melting point alloys can have a substantial influence on the obtained results of pull-out tests in small bone samples.

\section{Additional Reference}

Tsolaki IN, Madianos PN, Vrotsos JA (2009) Outcomes of dental implants in osteoporotic patients. A literature review. J Prosthodont 18: 309-323. 